\section{MALNUTRITION IN MEXICAN CHILDREN AND LEUKEMIA}

After careful reading of the article entitled: «Malnutrition, maintenance dose intensity and event-free survival in children with acute lymphoblastic leukemia» by Ibarra-Colado and Verdusco-Rodríguez ${ }^{1}$ we would like to acknowledge the signficant effort applied by these authors to the study of children with acute lymphoblastic leukemia and malnutrition as prognostic factor. However, we would like to comment several issues.

The authors refer that $20 \%$ to $50 \%$ of the children with acute leukemia present some degree of malnutrition. It is important to point out that, since the studies by Ramos-Galván ${ }^{2}$, it is considered that $60 \%$ of Mexican children present some degree of malnutrition; thus, in any third level institution, at least $50 \%$ of the regularly registered population shows different degrees of malnutrition. Therefore, it should be considered that $50 \%$ to $60 \%$ of the children with acute lymphoblastic leukemia at the National Institute of Pediatrics, or for that case, in any pediatric institution, present malnutrition. This indicates that the therapeutic results are lower than any international series, precisely because of the factor mentioned by the authors. However, our results with children of low economic level from rural and semi-rural areas and from larger communities subjected to rigurous chemotherapy protocols according to their risk classification are similar to those results published in industrialized countries $^{3,4}$. We therefore have to question where malnutrition is found as a prognostic factor.

Unfortunately, the series presented is too small to generate statistically significant results. In addition, the risk classification of these patients is incomplete, and no immunophenotype, cytogenetic or molecular biology study was obtained to determine the lineage and cytogenetic alterations as well as their molecular profile. We suppose that a minimal disease determination was performed, but it is not mentioned in the Results section. At present, this is considered a determinant prognostic factor.

Another debatable point is the chemotherapy scheme used. In the period of induction to remission the use of adriamycine or epirrubicine does not seem to be the treatment of choice with common risk. It has been demonstrated that in addition to vincristine and prednisone, L-asparaginase should be used to obtain a high rate of response and, in particular, more prolonged remission. Besides, no explanation is put forward for the use of cyclophosphamide with ara-C during consolidation, or why L-asparaginase was used in «others». Was there some kind of randomization, or was the combination chosen by chance? Maintenance seems to be conventional; however, in this type of leukemia it is not necessary to establish a three year program, since 30 months have been shown to be suf- ficient ${ }^{5}$. Finally, the disease-free period mentioned by the authors in well-nourished and malnourished patients is well below levels mentioned in the literature, which leads us to think that with a better structured and updated treatment the disease-free survival period would be longer.

In brief, it should be pointed out that the present study on malnutrition as a prognostic factor could not be documented for several reasons: it is not randomized, the number of patients in both arms is too small to determine statistically significant differences and multivariant analysis taking malnutrition as cardinal factor is absent.

\section{References}

1. Ibarra-Colado JE, Verduzco-Rodríguez L. Malnutrition, maintenance dose intensity and event-free survival in children with acute lymphoblastic leukemia. Rev Oncol 2002; 307-12.

2. Ramos GR, Mariscal AC, Viniegra CA, Pérez OB. Malnutrition in infants and children. Mexico DF: reports of the Hospital Infantil de Mexico; 1962;2. p. 18295.

3. Rivera Luna R, Cárdenas-Cardos R, Leal-Leal C, Navarro Alegría I, Meza-Coria C, Gómez-Martínez R. B-lineage acute lymphoblastic leukemia of childhood. An institutional experience. Arch Med Res 1997;28:233-9.

4. Riehm H, Gadner H, Henze G, et al. Results and significance of six randomized trials in four consecutive ALL-BFM studies. Heamatol Bluttransfus 1990;33:439-50.

5. George S, Aur R, Maeur A, et al. A reappraisal of the results of stopping therapy in childhood leukemia. N Engl J Med 1979;300:269-73.

\author{
Roberto Rivera-Luna ${ }^{\mathrm{a}}$ and \\ Rocío Cardenas-Cardos ${ }^{\mathrm{b}}$ \\ ${ }^{\text {a }}$ Subdirector of Hemato/Oncology. \\ ${ }^{\mathrm{b}}$ Head of the Oncology Service. \\ National Institute of Pediatrics. Mexico.
}

\section{AUTOR'S REPLY}

As we mentioned in our article, controversy exists on the role of malnutrition as prognostic factor in acute lymphoblastic leukemia. Malnutrition is a public health problem that persists in our country. Nevertheless the situation in Mexico has changed in the last 40 years. According to the National Survey of Nutrition of the SSA the prevalence of low weight (weight for age) at national level was of $7.5 \%$, the one of stunting (height for age) of $17.7 \%$ and the one of wasting (weight for height) of $2.0 \%{ }^{1}$. Prevalence of malnutrition in children with acute lymphoblastic leukemia in a Mexican third level hospital is as low as $3.8 \%{ }^{2}$. This contrasts with the 20\%-50\% reported in other hospitals of the country, obviously there is a reference bias in the population taken care in hospitals of third level.

The scheme of chemotherapy used in our population ${ }^{3}$ fulfilled the 1990 standards when our protocol began ${ }^{4}$. At the moment we used a scheme similar to the Total Therapy XIII of St. Jude ${ }^{5}$. Our experience is the one 